\title{
Nutrizione e ritmo circadiano: la nuova prospettiva del Time Restricted Feeding
}

\author{
Eleonora Poggiogalle ${ }^{1} \cdot$ Francesco Frigerio $^{1} \cdot$ Lorenzo M. Donini $^{1}$
}

Accettato: 9 novembre 2021 / Pubblicato online: 23 febbraio 2022

(c) The Author(s) 2022

\section{Sommario}

Negli ultimi anni il digiuno intermittente è emerso quale approccio innovativo per promuovere il calo ponderale e migliorare lo stato di salute metabolica in contrapposizione agli interventi più convenzionali incentrati sulla restrizione calorica. Il digiuno intermittente (Intermittent Fasting) e il Time-Restricted Feeding (TRF) negli animali (anche noto come TimeRestricted Eating, TRE nell'uomo) hanno raggiunto una crescente popolarità parallelamente al consistente aumento delle evidenze scientifiche nell'ambito della cronobiologia, con la recente attribuzione del premio Nobel per la Medicina nel 2017 a Jeffrey C. Hall, Michael Rosbash e Michael W. Young quale riconoscimento per il contributo alle scoperte dei meccanismi molecolari che sottendono il ritmo circadiano. Il sistema circadiano regola il metabolismo in un ciclo di circa ventiquattro ore, determinando una ritmicità circadiana endogena nel dispendio energetico, nell'appetito, nella sensibilità insulinica e in altri processi metabolici. Molti di tali processi, tra cui la sensibilità insulinica e la termogenesi indotta dalla dieta, presentano un picco nella prima parte della giornata. Numerose evidenze sia nell'animale sia nell'uomo sottolineano che alimentarsi in momenti della giornata che siano asincroni rispetto ai ritmi delle suddette funzioni metaboliche promuova l'eccesso ponderale e le alterazioni metaboliche ad esso associate.

Parole chiave Time restricted feeding · Intermittent fasting $\cdot$ Cronobiologia

\section{Il digiuno intermittente: un po' di storia}

La pratica del digiuno intermittente affonda le sue radici ante litteram nelle condizioni ambientali preesistenti rispetto alla rivoluzione industriale che ha determinato profondi cambiamenti nell'accesso al cibo sia in termini di introito energetico, sia nella frequenza di consumo di alimenti conducendo all' aumento progressivo dell'incidenza e prevalenza del sovrappeso, dell'obesità e delle patologie cardiometaboliche associate. L'avvento del lavoro notturno e il cosiddetto jet-lag sociale hanno ulteriormente messo a repentaglio la struttura cronobiologica che regola il funzionamento del nostro organismo [1], favorendo il consumo di cibo nel cosiddetto "buio biologico" e mostrando associazione epidemiologica con il rischio di obesità e di cancro (Box 1).

\begin{tabular}{l}
\hline Proposto da L.M. Donini. \\
\hline $\begin{array}{l}\text { E. Poggiogalle } \\
\text { eleonora.poggiogalle @ uniroma1.it }\end{array}$ \\
$1 \quad \begin{array}{l}\text { Dipartimento di Medicina Sperimentale, Sapienza Università di } \\
\text { Roma, Roma, Italia }\end{array}$
\end{tabular}

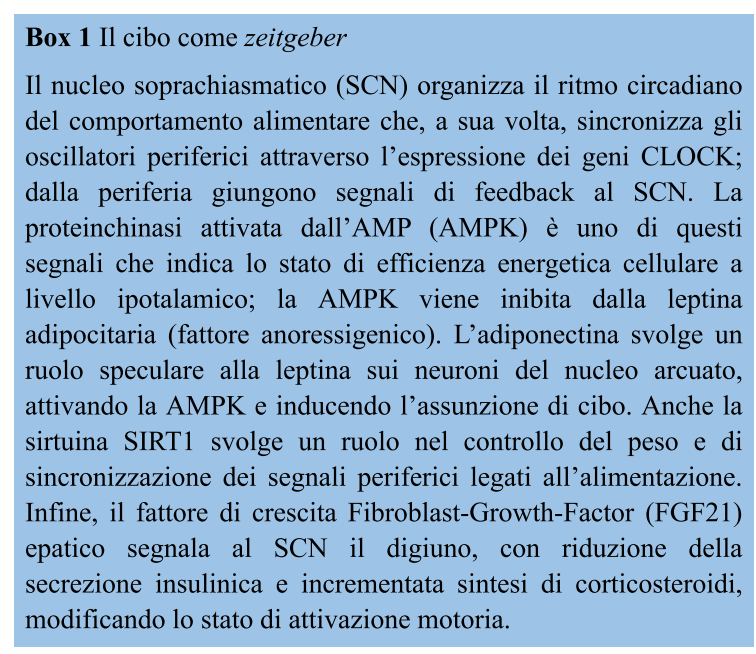

Se nelle ultime decadi la restrizione calorica (classicamente un deficit calorico di $500-1000 \mathrm{kcal} / \mathrm{die}$ o $75 \%$ del dispendio energetico totale giornaliero) ha dominato i tentativi di offrire soluzioni al sovrappeso e all'obesità, questi ultimi si sono rivelati scarsamente risolutivi nel medio-lungo periodo, con un recupero ponderale pressoché inevitabile nel 
corso del tempo a causa delle pressioni omeostatiche che regolano la composizione corporea, messe in atto in un ambiente obesogenico come quello contemporaneo [2]. Come mostrato da un recente ed esaustivo trial randomizzato controllato sulla restrizione calorica, soltanto il $50 \%$ della perdita ponderale veniva mantenuto nel follow-up a due anni dello studio CALERIE [3]. Per tali ragioni, la ricerca sul Time-Restricted Feeding ha ricevuto grande impulso con risultati preliminari incoraggianti, soprattutto alla luce della potenzialità di migliorare lo stato di nutrizione e di salute metabolica in assenza di riduzione dell'apporto energetico.

Alcune religioni, tra cui quella musulmana, offrono dei modelli di vita reale di digiuno intermittente, come avviene comunemente nella pratica religiosa del Ramadan. Milioni di musulmani aderiscono al Ramadan per una durata di circa 29-30 giorni nel mese "sacro", restando a digiuno dall'alba al tramonto e consumando la maggior parte dell'energia giornaliera immediatamente dopo il tramonto fino all'alba. Le riserve epatiche di glicogeno subiscono quindi una ciclica deplezione e replezione, mentre si verificano variazioni della miscela metabolica utilizzata nel corso della giornata, con progressivo incremento della quota lipidica ossidata verso il termine della giornata (e, quindi, del digiuno). Diverse meta-analisi hanno riportato un calo ponderale a seguito del Ramadan, con frequente recupero del peso nell'arco delle settimane successive (in particolare, tra due e cinque settimane dal termine dell'osservanza religiosa). Sia la massa grassa sia, seppur in misura minore, la massa magra sono risultate essere ridotte, così come pure la circonferenza addominale, in seguito alla pratica del Ramadan. Verosimilmente, la riduzione del compartimento adiposo è attribuibile all'aumento dell'ossidazione dei grassi durante la fase di digiuno. Per quanto riguarda, invece, gli effetti del Ramadan sulla tolleranza glucidica, le evidenze non sono consistenti né univoche a causa della variabilità delle metodologie utilizzate nonché della loro scarsa accuratezza per via del mancato utilizzo di tecniche gold-standard rivolte a valutare tali misure di esito (es. sensibilità insulinica).

\section{Intermittent fasting: un approccio scientifico}

Passando dal Ramadan a protocolli di Intermittent Fasting più strutturati e basati sull'evidenza, possiamo riassumere $\mathrm{i}$ diversi approcci in quattro strategie: Alternate-Day Fasting (ADF), Alternate-Day Modified Fasting (ADMF) e regime "5:2". Nella Tabella 1 sono indicate alcune delle principali caratteristiche di ciascun protocollo, basato sul digiuno completo (ADF) o modificato (ADMF, dieta 5:2) alternati a giornate di alimentazione libera, conducendo a un bilancio energetico settimanale negativo.

La strategia del digiuno intermittente propone di alternare periodi di digiuno (con una restrizione calorica variabile tra il 75 e il 100\%) a fasi di alimentazione ad libitum. Nell'ADF tre o quattro giorni a settimana richiedono il digiuno completo; il digiuno intermittente modificato (ADMF) consente l'assunzione del $25 \%$ delle calorie giornaliere in una finestra oraria ristretta, solitamente coincidente con un unico pasto, da ripetersi 3-4 volte a settimana. A livello mediatico ha guadagnato notorietà anche la dieta 5:2, con due giorni non consecutivi con apporto energetico non oltre il $25 \%$ del fabbisogno giornaliero che intervallano cinque giorni di alimentazione isocalorica.

\section{Dall'Intermittent Fasting al Time-Restricted Feeding}

I protocolli basati sul Time-Restricted Feeding (TRF) sono tra i più innovativi in quanto non prevedono la restrizione calorica né l'astinenza da cibo ma si basano sul prolungamento del digiuno giornaliero attraverso la restrizione della finestra oraria per l'accesso al cibo, senza modificare l'apporto energetico (Tabella 2). Numerose osservazioni in modelli animali suggeriscono marcati effetti positivi in termini di perdita ponderale, profilo lipidico, grasso ectopico, livelli di infiammazione, salute cardiologica, patologie oncologiche e prolungamento della durata della vita. Nell'uomo gli studi sul TRF hanno utilizzato finestre orarie di alimentazioni variabili, tra le 4 e le 12 ore, benché una finestra inferiore alle 10 ore sia ritenuta necessaria per elicitare un'ottimale modulazione della glicogenolisi, dell'ossidazione lipidica e della gluconeogenesi in assenza di disponibilità di glucidi di origine dietetica [4].

I due protocolli più studiati sono l'early- (eTRF) e il delayed-TRF (dTRF), nei quali gli interventi nutrizionali sono limitati rispettivamente alla prima o alla seconda parte della giornata. In misura minore è stato altresì applicato il cosiddetto midday-TRF, con l'intervento nutrizionale attuato nelle ore centrali della giornata. Studi preliminari senza gruppo di controllo hanno osservato un calo ponderale del $2-3 \%$ in un intervallo temporale variabile da uno a quattro mesi. In alcuni protocolli i partecipanti hanno aderito a un intervento di TRF, scegliendo una finestra oraria flessibile di otto-dieci ore nella quale alimentarsi ad libitum. Nonostante l'apporto calorico fosse distribuito in intervalli orari variabili nella fase diurna, la misura di esito del calo ponderale è stata raggiunta in maniera significativa sia negli adulti [5] sia negli anziani con sovrappeso o obesità [6]. L'evidenza da RCT suggerisce che, per quanto riguarda le misure di esito relative alla salute cardio-metabolica, i protocolli eTRF e midday-TRF abbiano prodotto dei risultati migliori rispetto alla finestra nutrizionale tardiva (dTRF). In uno studio cross-over che ha comparato l'eTRF al dTRF in uomini sovrappeso, entrambi i protocolli hanno indotto il calo ponderale e hanno migliorato i livelli di trigliceridi plasmatici e la tolleranza glucidica, mentre benefici sulla glicemia a 
Tabella 1 L'IF prevede giorni di digiuno completo (ADF) o modificato (ADMF, dieta 5:2) alternati a giorni di alimentazione libera per ottenere una condizione di bilancio energetico settimanale negativo. DET, Dispendio Energetico Totale

\begin{tabular}{lllllll}
\hline Protocollo & $\begin{array}{l}\text { Apporto } \\
\text { energetico giorni } \\
\text { liberi }\end{array}$ & $\begin{array}{l}\text { Giorni liberi }- \\
\text { finestra } \\
\text { alimentare }\end{array}$ & $\begin{array}{l}\text { Giorni digiuno }- \\
(\% \text { dispendio } \\
\text { energetico totale) }\end{array}$ & $\begin{array}{l}\text { Giorni digiuno - } \\
\text { finestra alimentare }\end{array}$ & $\begin{array}{l}\text { Giorni } \\
\text { alimentazione: } \\
\text { giorni digiuno (n:n) }\end{array}$ & $\begin{array}{l}\text { Restrizione } \\
\text { calorica }\end{array}$ \\
\hline ADF & Ad libitum & Libera & $0 \%$ & nessuna & $3: 4$ o 4:3 & Diretta \\
ADMF & Ad libitum & Libera & $25 \%$ & $2-4$ ore centrali & $3: 4$ o 4:3 & Diretta \\
$5: 2$ & Ad libitum & Libera & $25 \%$ & Libera & $5: 2$ & Diretta \\
\hline
\end{tabular}

digiuno sono stati osservati solo nel braccio eTRF attraverso il monitoraggio continuo della glicemia [7].

Tuttavia, alcuni autori hanno riportato un effetto metabolico deleterio nel caso della finestra alimentare tardiva ristretta al consumo di un unico pasto serale nell' arco di quattro ore [8] in partecipanti adulti normopeso. I benefici metabolici della finestra nutrizionale precoce sono stati osservati pur in assenza di calo ponderale in pazienti con prediabete, con un miglioramento significativo, a seguito di eTRF isocalorico, in termini di livelli di insulina, sensibilità insulinica, pressione arteriosa e stress ossidativo rispetto al gruppo di controllo che distribuiva l'apporto calorico sulla canonica finestra oraria di dodici ore.

Il primo RCT meccanicistico che ha esplorato la relazione tra effetti dell'eTRF, metabolismo energetico e variazioni dell'appetito, basato sull'utilizzo di metodiche goldstandard quali la camera metabolica, ha mostrato che l'eTRF non incide significativamente sul dispendio energetico totale giornaliero ma induce un incremento della termogenesi indotta dalla dieta e migliora la flessibilità metabolica, con importanti ricadute sull'ossidazione lipidica, stimolando la perdita di massa grassa e favorendo la perdita di peso senza ridurre l'apporto calorico [9]. Inoltre, i dati relativi alla regolazione dell' appetito hanno rivelato che l'eTRF è in grado di ridurre le concentrazioni di grelina, l'ormone che stimola la fame, soprattutto al mattino, e favorisce l'aumento dell'ormone della sazietà GLP-1. Dunque, l'ossidazione lipidica e la regolazione favorevole dell' appetito giocherebbero un ruolo cruciale nel promuovere il calo ponderale [9]. Nel medesimo studio è stato riscontrato un aumento del turnover proteico come conseguenza della gluconeogenesi nella fase di digiuno prolungato. Tale aspetto apre la questione della preservazione della massa magra durante un programma di calo ponderale. In uno studio basato sull'eTRF per dodici settimane è stata riscontrata una riduzione, seppur modesta, del compartimento muscolare. Un altro RCT ha invece dimostrato la sicurezza e la fattibilità del protocollo eTRF nell'ambito di individui non obesi in età adulta avanzata e in età geriatrica, scegliendo una finestra nutrizionale di circa otto ore per una durata di sei settimane, senza conseguenze deleterie sulla massa magra scheletrica e sulla densità minerale ossea [10]. Nell'anziano sarebbe dunque ottimale prediligere interventi eTRF di breve durata (quattro-sei settimane) con una finestra di digiuno meno estesa (quattordici-diciotto ore) [6].

\section{Midday-TRF e colazione}

Numerose campagne di educazione nutrizionale a livello nazionale e internazionale hanno conferito alla colazione il ruolo di "pasto più importante" della giornata. Tale posizione è stata messa in discussione dai più recenti studi di crononutrizione. Diverse meta-analisi hanno affrontato l'argomento con risultati discordanti. L'evidenza da studi trasversali o di coorte suggerisce un aumentato rischio di sovrappeso e obesità in caso di mancata colazione. A supporto di tale associazione sono stati identificati diversi meccanismi: l'aumento dei livelli circolanti di grelina che condurrebbe a un sovraconsumo compensatorio di cibo nella seconda parte della giornata, come pure delle alterazioni metaboliche sfavorevoli quali il peggioramento della sensibilità insulinica e l'aumento dei livelli di acidi grassi liberi circolanti in occasione del consumo del pranzo a seguito della colazione "saltata".

Anche l'espressione dei geni clock è influenzata dall'omissione della colazione, con effetti negativi in termini di glicemia postprandiale (sia dopo pranzo sia dopo cena), di ridotti livelli di GLP-1 e alterata risposta insulinica. Saltare la colazione indurrebbe l'iperattivazione indipendente dallo stress dell'asse ipotalamo-ipofisi-surrene (HPA), con alterazioni a carico delle concentrazioni del cortisolo plasmatico che sottenderebbero l'associazione tra il non fare colazione e patologie cardiovascolari (ad esempio, l'insorgenza di ipertensione arteriosa). Nel lungo periodo, l'assenza della colazione determinerebbe, invece, un pattern diurno "appiattito" del cortisolo, con oscillazioni diurne, indicative di una disfunzione dell'asse asse ipotalamo-ipofisi-surrene (HPA) che cronicamente può condurre a un peggioramento della salute cardiovascolare.

Altre meta-analisi di studi longitudinali riportano dati contraddittori, a favore di un' associazione tra mancata colazione e rischio di sovrappeso/obesità nelle persone che saltano la colazione per più di tre volte a settimana [11] o, al contrario, a favore di una modesta perdita di peso in caso di 
Tabella 2 Il TRF non prevede l'alternanza di giorni di digiuno e di alimentazione ma una finestra temporale giornaliera in cui alimentarsi. L'eventuale restrizione calorica è un effetto indiretto ottenuto dalla riduzione spontanea dell'apporto energetico durante la fase di ali- mentazione. In caso di alimentazione ad libitum è possibile che l'apporto energetico del paziente superi il suo dispendio energetico totale giornaliero (bilancio energetico positivo)

\begin{tabular}{llllll}
\hline Protocollo & $\begin{array}{l}\text { Apporto energetico }- \\
\text { finestra digiuno } \\
(\% \text { DET })\end{array}$ & $\begin{array}{l}\text { Apporto energetico }- \\
\text { finestra alimentare }\end{array}$ & $\begin{array}{l}\text { Restrizione } \\
\text { calorica }\end{array}$ & $\begin{array}{l}\text { Finestra } \\
\text { alimentare } \\
\text { giornaliera }\end{array}$ & $\begin{array}{l}\text { Esempi finestra } \\
\text { alimentare }\end{array}$ \\
\hline Early-TRF & $0 \%$ & Ad libitum & Indiretta & $<10$ h $(4-12)$ & 8 a.m. -5 p.m. \\
Delayed- TRF & $0 \%$ & Ad libitum & Indiretta & $<10$ h $(4-12)$ & 11 a.m. -8 p.m. \\
Religioso (Ramadan) & $0 \%$ & Ad libitum & Indiretta & $\begin{array}{l}\text { Tramonto-alba } \\
\text { (n. ore variabile) }\end{array}$ \\
\hline
\end{tabular}

colazione assente per un periodo di studio di circa due mesi, oppure senza mettere in evidenza il beneficio di introdurre la colazione perché questa pratica aumenterebbe l'apporto calorico giornaliero.

La nozione che la prima colazione sia necessaria per uno stile alimentare più sano risente dell'effetto confondente secondo il quale le persone che consumano abitualmente la prima colazione sarebbero più regolari nella distribuzione dei pasti e avrebbero uno stile di vita generalmente più salutare. Ulteriori evidenze hanno messo in relazione la mancata colazione con un aumentato rischio di diabete di tipo 2, pur correggendo l'associazione per $\mathrm{i}$ valori di indice di massa corporea [12]. Inoltre, in una meta-analisi che ha riportato un effetto positivo in termini di calo ponderale, l'assenza della colazione si associava a un peggiore profilo lipidico durante la giornata, in particolare con l'aumento del colesterolo "cattivo", LDL.

\section{Esercizio fisico e ritmi circadiani}

Anche il tessuto muscolare scheletrico mostra una ritmicità circadiana, con gli orologi periferici che determinano la risposta metabolica e l'efficienza dell'esercizio fisico. L'esercizio contro resistenza (es. esercizio isometrico, con pesi) è la forma di esercizio più suscettibile alle variazioni diurne, con il picco di forza muscolare tipicamente osservato tra il pomeriggio e la sera (16:00-20:00) e i valori minimi al mattino (06:00-10:00). Analogo pattern diurno è stato riportato per l'esercizio ad alta intensità [13]. La termoregolazione e le sue variazioni circadiane rappresentano una modalità indiretta attraverso la quale il nucleo soprachiasmatico influenza la risposta circadiana nel muscolo scheletrico e, indirettamente, l'efficienza della risposta all'esercizio fisico.

Le fluttuazioni circadiane della temperatura corporea sono responsabili della migliore efficienza contrattile muscolare nel tardo pomeriggio, così come dei processi metabolici (ad esempio, il turnover dell'ATP e il consumo di fosfocreatina) e della maggiore perfusione sanguigna tissutale.
Anche la funzione mitocondriale è regolata in senso circadiano: nei mitocondri isolati da biopsie muscolari nell'uomo, la capacità ossidativa aumenta alle ore 23 rispetto alle ore 04:00, 08:00, 13:00 e 18:00 [14]. Per quanto riguarda l'esercizio aerobico di lunga durata, come gli sport di resistenza, le evidenze sono controverse, con dati che comunque supportano una maggiore efficienza nella seconda parte della giornata. In conclusione, se svolti nella seconda parte del giorno biologico, l'esercizio aerobico e anaerobico hanno mostrato di modulare in modo clinicamente significativo il controllo glicemico il primo e la forza muscolare il secondo [15].

\section{Conclusioni}

Tali osservazioni suggeriscono che sincronizzare la finestra nutrizionale con il ritmo circadiano endogeno possa essere efficace nel ridurre l'apporto energetico quotidiano e ottimizzare il bilancio energetico, inducendo una maggiore flessibilità metabolica e migliorando la tolleranza glucidica e la composizione corporea. In conclusione, guardare l'orologio può essere più utile che guardare la bilancia [16].

\section{Glossario essenziale di cronobiologia}

- Acrofase (acrophase): il momento in cui un ritmo raggiunge il picco

- Allineamento (alignment): differenza di fase (allineamento) tra due ritmi qualsiasi

- Ampiezza (amplitude): misura di un'oscillazione come la metà della distanza tra zenith e nadir

- Angolo di fase (phase angle): intervallo di tempo tra la fase dell'orologio centrale (valutata dal DLMO) all'inizio della fase di sonno

- Dim Light Melatonin Onset (DLMO): comparsa della melatonina in prossimità della fase di buio biologico

- Entrainment: sincronizzazione di un ritmo a uno stimolo esterno o ambientale

- Fase (phase): il momento in cui si registra un punto stabile del ciclo, come un minimo 
- Loop a feedback trascrizione-traduzione (Transcriptionaltranslational feedback loop, TTFL): una serie di anse a feedback tra geni clock e proteine capace di mantenere ritmi di $24 \mathrm{~h}$ circa

- Mesor: valore medio di un ritmo

- Misalignment: anomalo allineamento (differenza di fase) tra due ritmi

- Nadir: il valore minimo assunto da un ritmo

- Periodo (tau, period): il tempo necessario perché l'oscillazione di un ciclo (es. un picco) si ripeta

- Picco (peak, zenith): il valore massimo assunto da un ritmo

- Ritmo circadiano (circadian rhythm): letteralmente "[della durata] intorno ad un giorno", si riferisce a un ritmo il cui ciclo completo si ripete ogni $24 \mathrm{~h}$ circa. È necessario misurare un ritmo in condizioni ambientali costanti (in assenza di zeitgeber) per dimostrare che esso viene generato endogenamente

- Ritmo diurno (diurnal rhythm): un parametro fisiologico che mostra cambiamenti durante il ciclo luce-buio così che picco e nadir avvengono a distanza di $12 \mathrm{~h}$ o in una frequenza armonica rispetto a $24 \mathrm{~h}$. In presenza di uno zeitgeber (es. il ciclo luce-buio), è impossibile distinguere se un ritmo sia generato endogenamente o sia una risposta acuta allo zeitgeber

- Spostamento di fase (phase shift): un cambiamento di fase di un ritmo che viene così anticipato (phase advance) $\mathrm{o}$ posticipato (phase delay)

- SCN: nucleo soprachiasmatico

- Zeitgeber: letteralmente "segna-tempo", qualsiasi stimolo che funge da segnale temporale dell'ambiente esterno. La luce e l'assunzione di cibo sono due zeitgeber rispettivamente per il SCN e gli oscillatori non-SCN.

Informazioni Supplementari La versione online contiene materiale supplementare disponibile su https://doi.org/10.1007/s40619-02201035-2.

\section{Dichiarazioni etiche}

Conflitto di interesse Gli autori Eleonora Poggiogalle, Francesco Frigerio e Lorenzo M. Donini dichiarano di non avere conflitti di interesse.

Consenso informato Lo studio presentato in questo articolo non ha richiesto sperimentazione umana.

Studi sugli animali Gli autori di questo articolo non hanno eseguito studi sugli animali.

Open Access This article is licensed under a Creative Commons Attribution 4.0 International License, which permits use, sharing, adaptation, distribution and reproduction in any medium or format, as long as you give appropriate credit to the original author(s) and the source, provide a link to the Creative Commons licence, and indicate if changes were made. The images or other third party material in this article are included in the article's Creative Commons licence, unless indicated otherwise in a credit line to the material. If material is not included in the article's Creative Commons licence and your intended use is not permitted by statutory regulation or exceeds the permitted use, you will need to obtain permission directly from the copyright holder. To view a copy of this licence, visit http://creativecommons.org/licenses/by/4.0/.

\section{Bibliografia}

1. Poggiogalle E, Jamshed H, Peterson CM (2018) Circadian regulation of glucose, lipid, and energy metabolism in humans. Metabolism 84:11-27

2. Dulloo AG, Jacquet J, Montani J-P, Schutz Y (2015) How dieting makes the lean fatter: from a perspective of body composition autoregulation through adipostats and proteinstats awaiting discovery. Obes Rev 16:25-35

3. Kraus WE, Bhapkar M, Huffman KM et al (2019) 2 years of calorie restriction and cardiometabolic risk (CALERIE): exploratory outcomes of a multicentre, phase 2, randomised controlled trial. Lancet Diabetes Endocrinol 7:673-683

4. Hoddy KK, Marlatt KL, Çetinkaya H, Ravussin E (2020) Intermittent fasting and metabolic health: from religious fast to time-restricted feeding. Obesity 28:S29-S37

5. Gill S, Panda S (2015) A smartphone app reveals erratic diurnal eating patterns in humans that can be modulated for health benefits. Cell Metab 22(5):789-798

6. Anton SD, Lee SA, Donahoo WT et al (2019) The effects of time restricted feeding on overweight, older adults: a pilot study. Nutrients 11:1-9

7. Hutchison AT, Regmi P, Manoogian EN et al (2019) Timerestricted feeding improves glucose tolerance in men at risk for type 2 diabetes: a randomized crossover trial. Obesity 27(5):724-732

8. Carlson O, Martin B, Stote KS et al (2007) Impact of reduced meal frequency without caloric restriction on glucose regulation in healthy, normal-weight middle-aged men and women. Metabolism 56:1729-1734

9. Ravussin E, Beyl RA, Poggiogalle E et al (2019) Early timerestricted feeding reduces appetite and increases fat oxidation but does not affect energy expenditure in humans. Obesity 27(8):1244-1254

10. Martens CR, Rossman MJ, Mazzo MR et al (2020) Short-term time-restricted feeding is safe and feasible in non-obese healthy midlife and older adults. Geroscience 42:667-686

11. Wicherski J, Schlesinger S, Fischer F (2021) Association between breakfast skipping and body weight - a systematic review and meta-analysis of observational longitudinal studies. Nutrients 13:1-20

12. Mekary RA (2019) Breakfast skipping and type 2 diabetes: where do we stand? J Nutr 149:1-3

13. Mancilla R, Krook A, Schrauwen P, Hesselink MK (2020) Diurnal regulation of peripheral glucose metabolism: potential effects of exercise timing. Obesity 28:S38-S45

14. Gutierrez-Monreal MA, Harmsen JF, Schrauwen P, Esser KA (2020) Ticking for metabolic health: the skeletal-muscle clocks. Obesity 28:S46-S54

15. Parr EB, Heilbronn LK, Hawley JA (2020) A time to eat and a time to exercise. Exerc Sport Sci Rev 48:4-10

16. Vaughan KL, Mattison JA (2018) Watch the clock, not the scale. Cell Metab 27:1159-1160

Nota della casa editrice Springer Nature rimane neutrale in riguardo alle rivendicazioni giurisdizionali nelle mappe pubblicate e nelle affiliazioni istituzionali. 